\title{
MANAGING INTEGRATION TECHNOLOGY OPTIONS: DATAPOWER AT WOOLWORTHS SOUTH AFRICA ${ }^{1}$
}

Tshepo Manaka, an analyst programmer in the integration team at Woolworths Holdings, South Africa, was responsible for development and configuration of the DataPower appliance. The DataPower appliance was used for integration of data between Woolworths South Africa and external units; it worked as a multi-protocol gateway. Three months after the integration of Woolworths South Africa with the Australian retailer David Jones, Tshepo observed that there were frequently large volumes of data being transferred at Woolworths that were causing low DataPower disk space resulting in integration issues and violations of the Service-level Agreement. He had to figure out how the diminishing of the DataPower disk space could be resolved.

Woolworths South Africa was one of the biggest retail companies in South Africa. It sold products to the high-end market in South Africa. It faced multiple challenges that began when they acquired an Australian retail company, David Jones. There were framework changes in order to integrate the companies and there were also challenges of integration of the two companies' systems. With respect to the DataPower application, the main issue faced by the company was the diminishing of disk space. As a multi-protocol-gateway, DataPower was used to receive data from a client source in various protocols after which it had sent the data to a target site using various protocols.

In making his decision, Tshepo had six options he could potentially choose from to address the problem at hand: 1) carry out configuration of DataPower so that it was optimized to cater for the heavy load of data; 2) carry-out a firmware upgrade to DataPower; 3) purchase a new DataPower device with a higher disk space; 4) optimize the applications that used DataPower to streamline the data, thus reducing the load on the DataPower device; and 5) use the secondary DataPower device to balance the load with the primary appliance. The last option was to use a combination of the options stated above.

All options had the potential to help with the problem of disk space of DataPower but there were a number of constraints affecting all the options, ranging from time, personnel, funding as well as information needed to implement the solution. The integration team was the final decision maker for implementations, using the options that were presented by Tshepo. Ultimately, the implemented solution needed to be reliable for the coming years, since the load of data transfers was expected to continue to increase owing to the demands of the business.

\footnotetext{
${ }^{1}$ Copyright (C) 2017, Tshepo Manaka. This case was prepared for the purpose of class discussion. Names and some information have been disguised. This case is published under a Creative Commons BY-NC license and originally appeared as a chapter in Transforming Society Using ICT: Contemporary Discussion Cases from Africa. Permission is granted to copy and distribute this case for non-commercial purposes, in both printed and electronic formats.
} 


\section{South Africa and South African Retail}

South Africa was located at the southernmost tip of Africa. It had a long coast line that stretched from the Atlantic to the Indian Ocean. It was home to some of the biggest and the most visited cities in Africa, namely Johannesburg and Cape Town. The country was rich with heritage and boasted 11 official languages. It had many natural resources, including minerals such as gold and diamonds. The wildlife in South Africa was diverse and included the "big 5" of the animal kingdom in the local nationals parks, the most famous being the Kruger National Park.

South Africa was considered to be in the developing country category and had opportunities and the potential to be a world class country. Economically, it had a wide gap between the lower class and the upper class income levels. Many of its poor citizens lived well below the poverty line, while many of its rich were extremely rich. The South African economy had generally been stable but, in recent years, had been going through rough patches mainly due to the politics of the government. The political challenges started facing the country were largely derived from the Apartheid era, where an all-white government had been suppressing and discriminating against the non-white (black) people of South Africa. The black people of South Africa had been separated from the whites in all areas of society such as transport and common areas like toilets. They also had only been granted limited access to education-education being conducted in a language that was not theirs.

In the early 90s apartheid was overthrown and the people of South Africa were granted equal rights to vote and live in South Africa. Opportunities arose during the post-apartheid era for black South Africans, from education and up-skilling to establishing businesses. In this era big retailers were established and gained traction in the South African market, making it one of the biggest contributors to the country's economy.

\section{Retail in South Africa}

South Africa was one of the giants in the retail market of Africa, and the 20th largest in the world. There were a handful of companies that dominated the country's industry, with the majority of brands being owned by them. The structure of the industry moved towards mall-based retailing, for reasons including security and accessibility. Along with the dominant companies there were a large number of small to medium retailers, many of which were situated in the informal settlements. The large number of South Africans living below the poverty line gave rise to these retail shops, also known as 'spaza' shops, which catered for their basic needs. The economy was strongly impacted by the uncertainty in the global marketplace, sometimes resulting in substantially decreased retail sales. Contributing factors to the volatility of the retail marketplace included inflation, as well as increasing costs of fuel and electricity (WRLC, 2013) (See Exhibit 1).

At the time of the case, the country's monthly trade sales had been rising since late 2009 from less than 47 billion rand to over 59 billion (StatsSA, 2015). Even though the retail industry trade sales were on an incline, there was a decline in the retail sales growths from $6.1 \%$ in 2011 to $2.4 \%$ in 2014.

The declining growth rate made retailers aware that a change was required to keep the momentum going if retail sales were to remain on the incline. Retailers took different approaches to increasing sales growth. The first approach was expand the franchises beyond the borders of South Africa. The second was to import goods from other countries where production costs were cheaper than those in South Africa, allowing them to be sold at a cheaper price. The third approach was to merge companies together to make a bigger retailer. Finally, other retailers decided to target a certain market where they expected to get more sales. 
Woolworths' approach had been to acquire the Country Road group and David Jones, thereby expanding beyond South African shores. The acquisition helped Woolworths become one of the biggest retailers in the Southern hemisphere, extending its branches in over 11 countries in Africa and across the ocean to Australia and New Zealand.

Through the growth of the retail industry in South Africa, there had been a growing need to implement information technology (IT) solutions that analyzed market trends in order to plan for the future. Retail involved the sharing of a huge amount of data, such as sales, customer information and inventory, in order to better manage operations and marketing. Even though IT represented a relatively small percentage of the retail industry's costs, companies could not function without it.

\section{IT Architecture Concepts}

The challenges faced by Tshepo derived from the complexity of its IT architecture. Among the concepts involved were those of middleware, integration and the implementation of an enterprise service bus.

\section{Middleware}

According to the Woolworths' application principles and guidelines, middleware was known as a set of common business-unaware services that enabled applications and end-users to interact with each other across a network. Middleware gave the ability to integrate the diverse data and information sources from inside and outside the organization into a single logical framework.

The primary function of middleware was to minimize the changes to the existing applications by providing a flexible formatting and transporting layer in order to deliver data in a format that the target system could handle. This meant that every transfer of data should go via the middleware. Middleware was not to be used to perform business calculations or enrich data, thereby minimizing network traffic and avoiding bottlenecks of data being transferred. The use of middleware should not be used primarily for backup or a staging area for data, but middleware will archive the data after a number of days or a couple of weeks depending on how it was setup.

Middleware was able to monitor transactions in order to perform commits and rollback if there were any issues. If the data was lost during the transaction, the middleware should be able to re-send the data to the target system. Middleware can be seen as some sort of proxy that acts as an intermediary for transferring data from the source system to the target system.

\section{Integration}

Integration was known as the process of putting system components or applications together into one system and making sure that the system components work together. Integration makes the different systems in an organization work together and allows for seamless incorporation within the organization. In other words it was the glue that held the organization's systems together in order for them to function effectively.

Within an organization there are multiple applications used between the different departments. These departments' applications exist on different platforms and coded in different programming languages using different data formats. Applications use different protocol to transfer data to other departments. Integration was a solution between the organizations applications to transfer data and it allows organizations to cut costs and resources. Integration acts like a switchboard whereby no single application 
will connect directly to another, but via switchboard that simplifies multiple connections. This switchboard had multiple adaptors that allow applications to connect to the switchboard itself and from the switchboard to other applications. Integration does not only route data but also filters, transforms and enriches data.

\section{Enterprise service bus (ESB)}

ESB was a framework for middleware that provides services to cater for complex architectures. Access to application and services were managed by ESB in order to provide a single, simple, and consistent interface to end users. ESB allowed connection of multiple systems, applications and data without needing to write customized programs to integrate the different systems. If one system changed, the other systems were not affected since the connection would only be changed on the ESB while the other systems remained the same (See Exhibit 2 \& 3).

The purposes of ESB purposes included:

- Fast and easy delivery of information across an organization.

- Platforms, software architectures and network protocols differences to be masked.

- Regardless of systems or networks going offline, information was guaranteed to be delivered.

- Information could be re-routed, logged and enriched without requiring applications to be reworked.

- Avoiding the need for the organization's services applications to change immediately or all at once.

\section{Message Broker}

Message Broker was an IBM product that was also known as Integration Bus. It was a program module that was used to retrieve data from application within an organization via a formal messaging protocol, transforming the data or messages into a format or data structure acceptable by the target application then sending the data to the target in various formal messaging protocols. Message Broker acted as middleware, integrating the systems of the organization. It also monitored the transaction data and was able to respond to events and errors.

Message Broker supported a wide range of protocols such as File, WebSphere MQ, HTTPS, Web Services and JMS. According to IBM, the operations that Message Broker supported included routing, filtering, monitoring, transforming, enriching, collection, detention, distribution and correlation.

IBM Message Broker was used in Woolworths in the integration space to ensure that there was seamless exchange between systems running at the Head Office, in stores, distribution centers and at suppliers. No systems within Woolworths should communicate directly; therefore everything was supposed to go through Message Broker (See Exhibit 4).

\section{DataPower}

DataPower, also an IBM product, was an appliance or a rack mountable device that was pre-built and preconfigured. It served as a multi-channel gateway that helped with the integration of an organization with its external partners, ensured the security of the data being transferred, controlled data traffic and monitored performance to ensure compliance with service level agreements. It was also known to be an $\mathrm{xml}$ processing engine that accelerated the processing of $\mathrm{xml}$ - the text-based format widely used in data exchange over the internet. Drilling down to its functions, the multi-channel gateway was a gateway that received data, and routed it to the target site using the application's protocol. Protocols supported included FTP, HTTPS, AS2 and more (See Exhibit 5). 
The security component of DataPower had capabilities that incorporated decryption and encryption of data transferred over the network. DataPower had fine grained Authentication, Authorization, and Auditing features that ensured the right permissions were set to perform certain tasks. It extends the Service-oriented architecture (SOA) infrastructure and form part of the integration layout (See Exhibit 6).

DataPower was licensed by IBM under terms and conditions that specified warrantee, life span, services and support. DataPower made appliance service and support available for 5 years. Prior to the discontinuination of the support for the appliance, a notice was published 12 months before the effective date (IBM, 2016). DataPower provided firmware releases every 2 years that incorporated bug fixes and upgrades.

Unless the appliance and firmware upgrades were installed, DataPower would not be supported after a specified period of time. Without the upgrades, the appliance was likely to have unfixed bugs and its performance was expected to deteriorate. Organizations were expected to be aware of DataPower's life cycle (See Exhibit 7).

\section{Woolworths Holdings Limited}

Woolworths holding limited was a South African retailer and one of the top 5 biggest retailers in South Africa with close to \$4 950 million in retail revenue (Case Writer, 2016). Woolworths was modeled after British retailer Marks and Spencer. The products sold ranged from luxury foods to premium clothing. The target market of Woolworths was from the middle to the upper class of the Southern African market. The Woolworths brand was known for having quality and healthy products but came with a hefty price tag. Woolworths was also called 'woolies' by the local people. Their slogan was 'the difference'.

Woolworths South Africa consisted of several departments: Woolworths Clothes and General Merchandise, Food and Financial Services. The departments formed part of a Woolworth's group under Woolworths Holding Limited, which included David Jones and Country Road Group under its umbrella as well (Woolworths LTD, 2014) (See Exhibit 8).

The values that Woolworths established a foundation for the business were Quality and Style, Value, Service, Innovation, Integrity, Energy and Sustainability, further explained in Table 1 of Exhibit 9. The values were used to give the company direction, and to guide "behavior, actions and choices".

Woolworths' internal structures were headquartered in Cape Town. The structures include Clothing and General Merchandise, Foods, Marketing, People, Information Technology, Real Estate Development, Facilities and Corporate Governance (Woolworths, 2014). The company's external structure included over 170 stores, distribution centers and suppliers (see Exhibit 10).

\section{Middleware at Woolworths}

Making effective use of technology became particularly difficult when Woolworths merged with other retailers, creating the need to integrate the technologies of the merging companies. Substantial complexity was introduced when merging the propriety data held by each company. Not only did different companies store different types of data, they also did so in different formats and across different periods. One of the best ways to handle this varying of the propriety format of data was to use middleware between the merged companies. This middleware served to allow existing proprietary networks to translate and transfer data from one network to another. 
The middleware layout of Woolworths used IBM products. WebSphere, more specifically DataPower, handled most data traffic in and out of applications and between partners. Woolworths' DataPower devices were 4 years old and had been configured to be flexible in order to support adding new services and configurations. During the acquisition of the Australian retailers, however, there were complex integration challenges ranging from the technology used by each party and the huge amounts of data. Woolworths was the mother company, meaning it had to accommodate these integration challenges.

Without getting new infrastructure, new services and configurations of the middleware-which included Message Broker and DataPower-were implemented to cater for the demand. In the Message Broker area there were a lot of transformations and data enhancements that had to be made to integrate all applications, leading to the use of more resources in order to process the large volumes of data. In theory, DataPower could handle the load because DataPower was not a storage device but was, in fact, a transmission device. The system's disk space was used for configurations and for the run time environment. However, due to the high volumes of data being transferred through DataPower, the amount of disk space being used kept increasing. As a result, when the capacity was reached the device either hung or had to be restarted in order to free up some space. This process resulted in some of the transferred data failing as well, since some of the data got lost during the restart. This resulted data in not being delivered at the required time, a violation of the company’s Service Legal Agreement (SLA).

\section{Application Integration Team (AIT)}

AIT was the Woolworths integration team that provided seamless integration solutions between systems running at Head Office, insStores, distribution centers and at suppliers. No systems within Woolworths were supposed to communicate directly; everything was expected to go through AIT. AIT provided integration for systems running in all areas of IT - Foods, Clothing \& Home, In the Bag, Woolworths Financial Services, Human Resource, Finance, Logistics, Stores, Planning, and Information Delivery.

The AIT team consisted of groups that functioned together in the integration space. The Automation and scheduling team, Interface Development team, Infrastructure team and the Supporting Technology team were the main groups within the team. They all had specific roles in the AIT team but came to work together at the end for the solution. Within the Supporting Technology team there was a DataPower team where Tshepo was a core member. He dealt with all the DataPower developments from projects as well as day-to-day operations and enquiries.

Within AIT, the following layers of decision makers were in finding a solution to decreasing disc space untilization in the DataPower device: AIT managers, AIT infrastructure/architectures, Supporting technology manager and the Analyst programmer. These are illustrated in Figure 1 of Exhibit 11.

The AIT Management was a group of AIT managers in the different AIT structures that were responsible for the bigger AIT decisions. All decisions by AIT had to be approved by them as they were responsible for allocating the resources and finances for the project to be approved. The second layers of the decision making were the AIT infrastructure or architecture group, which was responsible for all the designs and architecture of systems in AIT. They determined how the systems fit into each other, creating infrastructure that would be effective and efficient for the purpose of an optimal integration space.

Third on the decision making hierarchy was the Supporting Technology (ST) manager, who was responsible for decisions relating to the product itself - DataPower. The ST Manager was the most important decision maker with regards to the specific system requirements and the need to change the appliance. Basically, they served as the 'owners' of the product. Lastly at the bottom of the hierarchy was the analyst programmer who used the products, responsible for setup, configurations and development of services. 


\section{The Decision}

Tshepo had investigated various options available to solve the problem of DataPower's disk space reaching the maximum capacity. The low disk space had already caused problems, since it had resulted in the DataPower device restarting, causing transactions to fail and lose data. Unless addressed, these issues would violate the business SLA by failing to deliver all data to the target systems, resulting in the loss of vital data, such as payroll and sales information. Not finding a solution and fixing the problem would reduce Woolworth's IT integration reliability, as well as negatively impacting its reputation. In addition to these consequences, Woolworths would lose a lot of money due to mismatching of data and the recovery of the data that was lost due. After investigating the situation, Tshepo uncovered several alternative approaches to the problem. These included the following options.

\section{DataPower Optimization}

The first option was to optimize DataPower services and configurations so that the appliance performed better. This decision would require analysis and evaluation of services by checking where the problem areas were. This would require considerable effort from Tshepo and the ST Manager, even though both already worked closely with DataPower and had considerable knowledge relating to how the systems worked and were configured. The optimization would free up some disk space and increase transfer speeds if done optimally and correctly. From the AIT financial point of view, this would be the cheapest choice as no external interventions were required and all the resources needed were onsite. There were, however, likely to be significant limits with respect to the performance benefits that could be obtained solely through reconfiguration. Moreover, performance could easily degrade again in the future as demands on the system increased.

\section{DataPower Firmware upgrade}

Firmware upgrade was the second option. An upgrade from the existing firmware was already available for the current device. That upgrade reportedly included optimizing the DataPower framework and fixing exiting bugs. Tshepo speculated that it would work but only for certain issues that had already been raised with IBM. The risk was that the bug fixes or optimization might not have any effect on the Woolworths issue. Worse, like all major system upgrades, it had the potential of causing existing applications that worked property to fail. Even though it was viewed as a good option, the new firmware had to be analyzed to see what was being fixed or changed so that it did not affect the system negatively - a process that could take a considerable amount of time and effort.

\section{New DataPower Appliances}

The third and the most expensive option was to get a new DataPower appliance. This would require all resources to be involved as the migration process would include a lot work. The appliance would be placed at the Woolworth's data center (DC) and all network cables would have to be connected. All the services and configuration would need to be migrated from the old to the new. Tshepo would need to ensure that thorough testing was conducted to ensure that the components were working successfully. Only then could the new devices be switched on, at which point all the existing service would be running on the new device. 


\section{Other Applications Optimization Systems}

Optimizing the systems connecting to DataPower was another option on the table. Systems such as Message Broker, Managed file transfer (MFT), Message Queue (MQ) infrastructure could be analyzed, optimized and reworked in order to help DataPower with the low disk space issue. For example, these systems could be reconfigured to send chunked messages, which were big messages that are cut into smaller pieces and passed through DataPower. This would help with memory consumption and, hopefully, the transfer speeds. The solution would require many resources because it was not only related to DataPower but all the systems around it. Testing would also be a big factor as there would be many test scenarios to check if the changes had been successful in combating the low disk space issues.

\section{Load Balance with Disaster Recovery DataPower}

The last option considered was the use of Disaster Recovery (DR) DataPower, a backup system put in place to allow continuity of operations in the event that the primary appliance failed, to load balance with the primary appliance. This process would shed some load on the disk space of the primary by sharing the load with the DR DataPower. The only thing required was to ensure that both appliances were identical with respect to services and configurations. This would be followed by the networks team routing the transactions to both appliances equally. This option was actually part of the future architectural setup that had been suggested by the infrastructure/architectural team.

\section{Parting Thoughts}

The options needed to be evaluated in terms of cost to the company, resources available, time consumption, and the likelihood that the selected option would fix the problem. Tshepo would then propose the best option, or a combination of options, to his supervisors. Once a decision was made, a review meeting had to be held with the rest of Woolworths' IT to determine any threats and opportunities the option may bring about. What made this particularly challenging was the fact that Tshepo felt that the option most certain to solve the problem immediately_buying a new DataPower device—also happened to be the most expensive and time-consuming. Thus, from a business point of view, all other options had to be explored.

\section{References}

WRLC. (2013). Retail Business in South Africa. Retrieved July 01, 2017, from http://wrlc.org.za/retaillandscape-2/retail-business-in-south-africa/

StatsSA (2015, February 18). Retail sector growth losing steam. Retrieved July 01, 2017, from http://www.statssa.gov.za/?p=4163

WOOLWORTHS HOLDINGS LIMITED. (2014, April 9). Woolworths South Africa to acquire David Jones to create a leading southern hemisphere retailer [Press release]. Retrieved July 01, 2017, from http://www.woolworthsholdings.co.za/whl_mini_2014/pdf/press_release_final.pdf 


\section{Author}

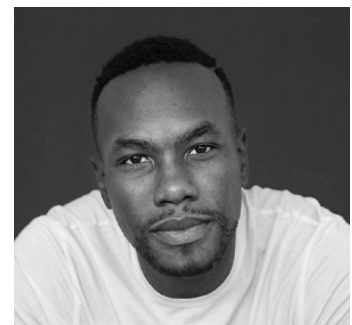

Tshepo Manaka is an Analyst Programmer at Woolworths Holding in South Africa. He holds a BA degree in Human Movement Science from the University of Pretoria. He has received a Postgraduate diploma in Information and Communication Technology from the University of the Western Cape. He recently completed a Postgraduate diploma in Management: Information Systems at the University of Cape Town. 


\section{Exhibit 1: Retail Business in South Africa}

6772017

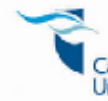

Cape Peninsula
University of Technology

\section{Wholesale \& Retail Leadership Chair}

"Collaboration opens the window to a world of opportunities"

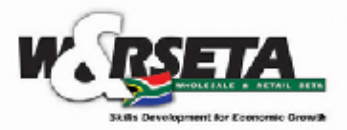

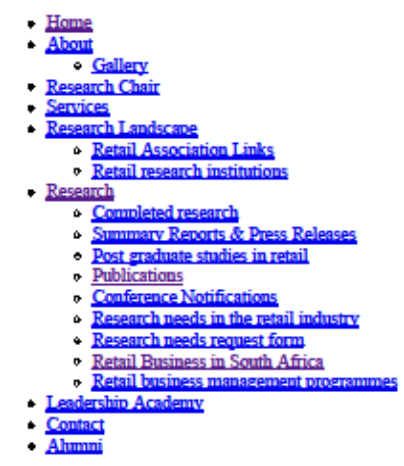

Retail Business in South Africa

The South African retail market is a giant compared to the rest of Africa, but also the 20th largest in the world. In 2011 retail sales for the first time in history surpassed the one trillion rand mark. Yet the retail market is dominated by only about a dozen large holding companies which collectively own the majority of the country's biggest brands. The biggest of these. Shoprite, Pick n Pay, Spar and Massmart - account for about $80 \%$ of local retail sales. With the exception of Massmart, which was taken over in 2011 by Wallmart, the world's largest retailer, all the others are South African ouned Because of the dominace of the larger players, the Soun African resil medium and small operations, of which a significant portion consists of informal traders. In fact, the lower end of the scale is characterised by about a 100 opo informal spaza shops with an estimated in this sector Volume African rail indrty is the way bow retriers are peshaps leading the second scramble for Africa.

Copyright O WRLC 2013. All Rights Reserved

Source: http://wrlc.org.za/retail-landscape-2/retail-business-in-south-africa/ 


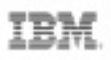

\section{IBM DataPower - Converged Multi-Channel Gateway}

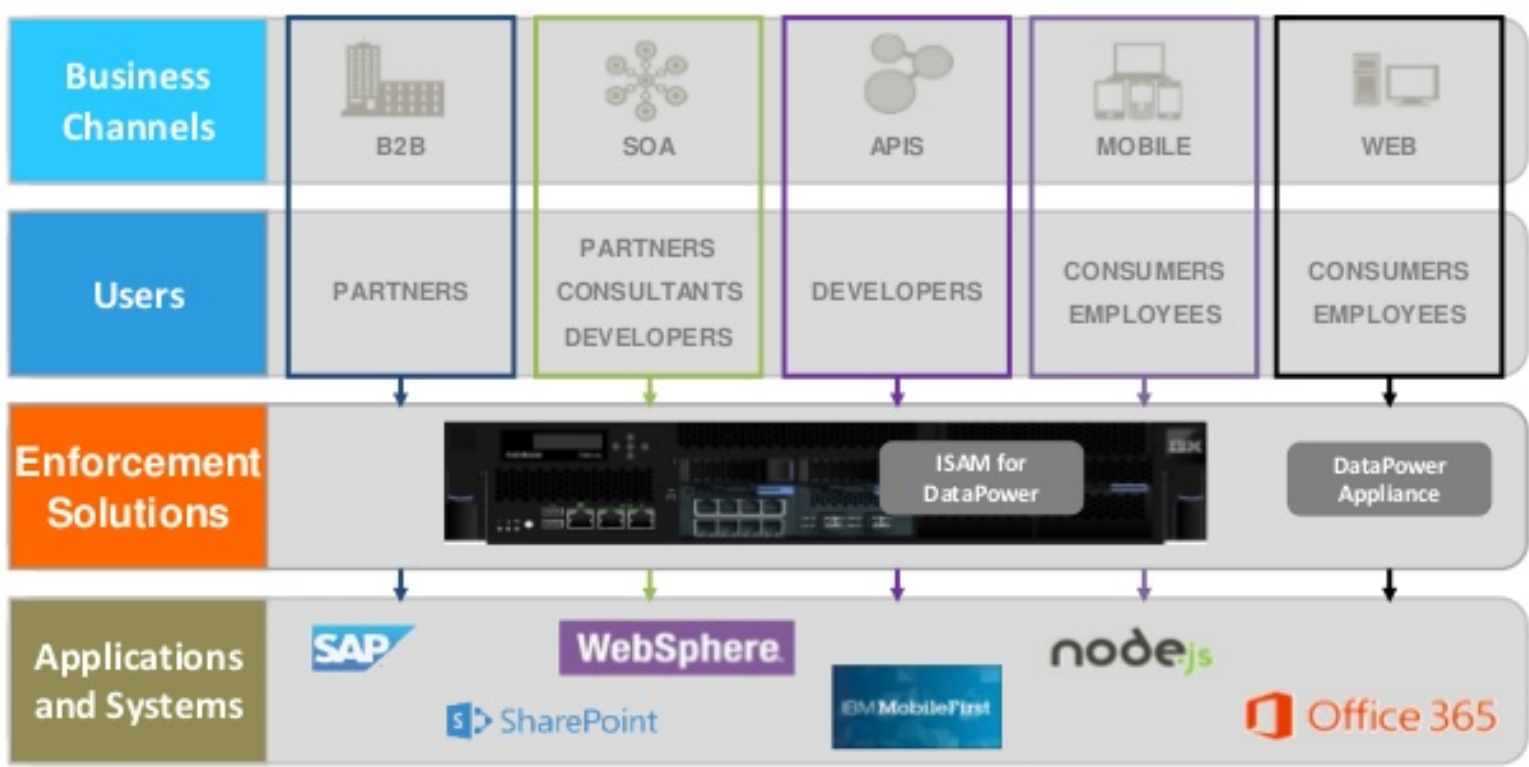

Source: https://www.slideshare.net/Valerilllescas/datapower-steven-cawn 


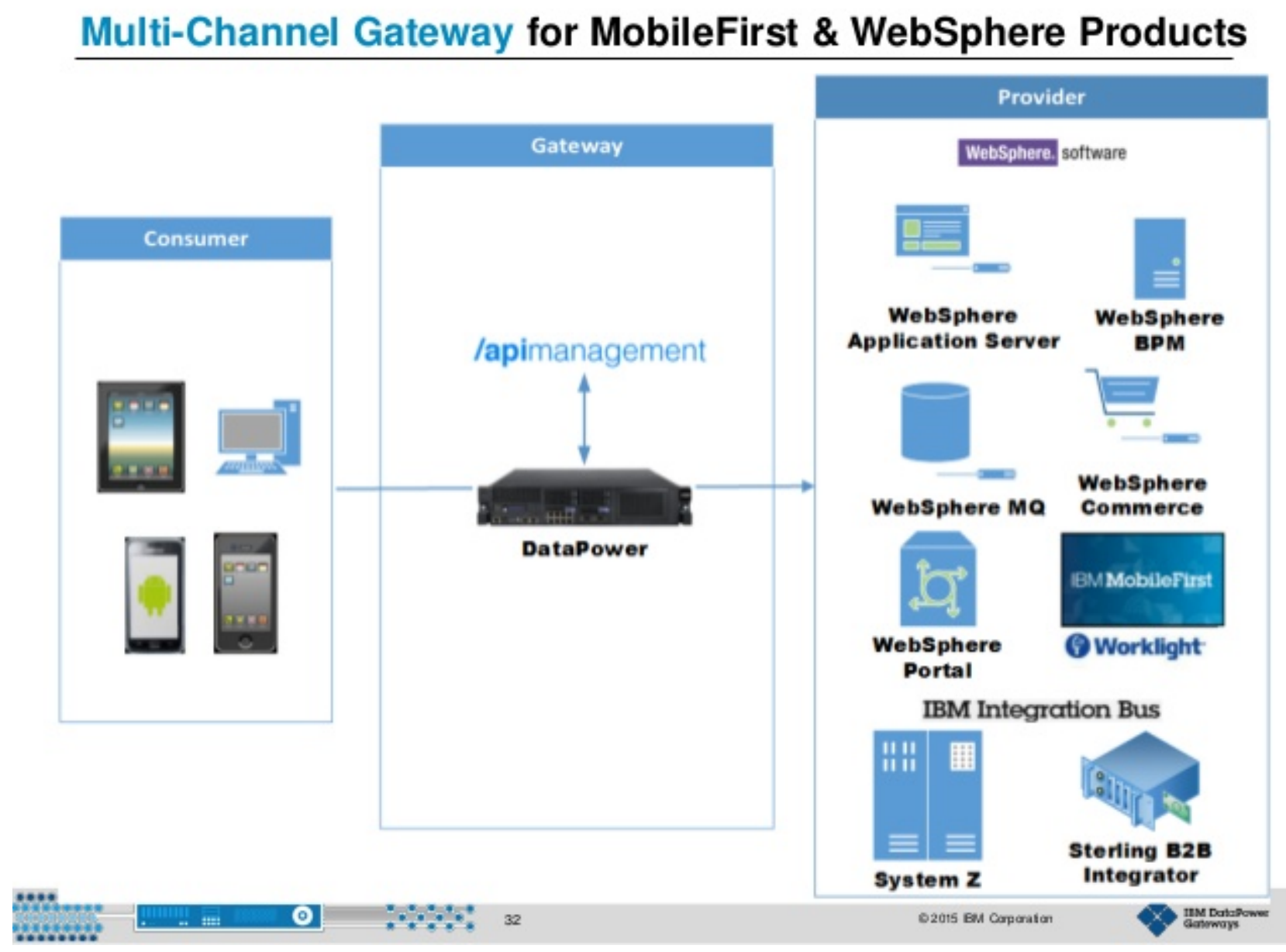

Source: https://www.slideshare.net/albertspijkers/secure-and-optimize-delivery-of-multi-channelservices-using-ibm-data-power-gateways 
MUMA CASE REVIEW

\section{Exhibit 4: Message Brokerl Integration Bus}

$67 / 2017$

IEM Integration Bus - WIKIpeda

\section{IBM Integration Bus}

From Wikipedia, the free encyclopedia

IBM Integration Bus (formerly known as WebSphere Message Broker) is IBM's integration broker from the WebSphere product family that allows business information to flow between disparate applications across multiple hardware and software platforms. Rules can be applied to the data flowing through the message broker to route and transform the information. The product is an Enterprise Service Bus supplying a communication channel between applications and services in a service-oriented architecture.

IBM Integration Bus provides capabilities to build solutions needed to support diverse integration requirements through a set of connectors to a range of data sources, including packaged applications, files, mobile devices, messaging systems, and databases. A benefit of using IBM Integration Bus is that the tool enables existing applications for Web Services without costly legacy application rewrites. IIB (IBM Integration Bus) avoids the point-to-point strain on development resources by connecting any application or service over multiple protocols, including SOAP, HTTP and JMS ${ }^{[1]}$ Modern secure authentication mechanisms, including the ability to perform actions on behalf of masquerading or delegate users, through MQ, HTTP and SOAP nodes are supported such as LDAP, X-AUTH, O-AUTH, and two-way SSL.

IBM Integration Bus embeds a Common Language Runtime to invoke any .NET logic as part of an integration. It also includes full support for the Visual Studio development environment, including the integrated debugger and code templates. IBM Integration Bus includes a comprehensive set of patterns and samples that demonstrate bi-directional connectivity with both Microsoft Dynamics CRM and MSMQ.

\section{Contents}

- 1 Features

- 2 History

- 3 Components

- 4 How IBM Integration Bus works

- 4.1 Overview

- 4.2 Market position

- 4.3 Expected performance

- 4.4 Message flow nodes available

- 4.4.1 Message flow node types

- 4.5 Localization

- 4.6 Patterns

- 4.6.1 Pattern examples

- 5 Supported platforms

- 5.1 Operating systems

- 6 See also

- 7 References

- 8 External links

\section{Features}

Source: https://en.wikipedia.org/wiki/IBM_Integration_Bus 
MANAKA

Exhibit 5: DataPower Explained

Product Announcement

March 28, 2006

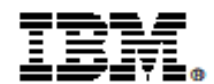

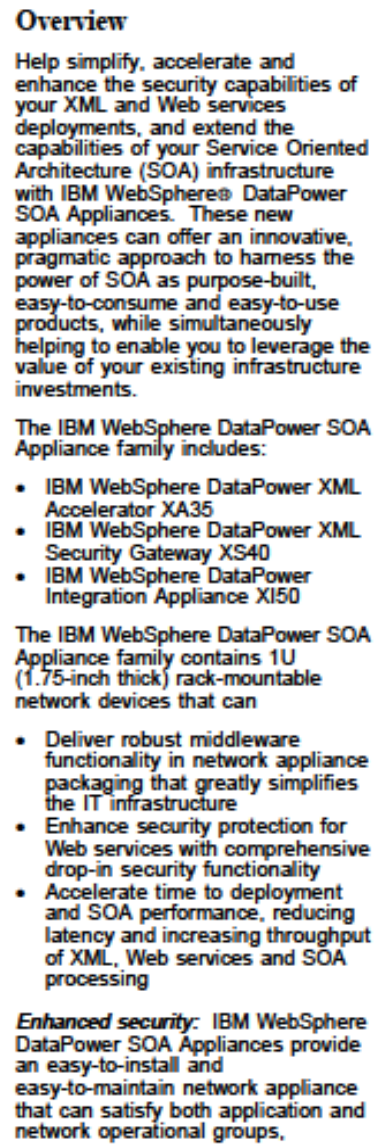

Overview

Help simplify, accelerate and enhance the security capabilities of your XML and Web services capabilities of your Service Oriented Architecture (SOA) infrastructure with IBM WebSpheres DataPower with IBM WebSpherea DataP
SOA Appliances. These new appliances can offer an innovative, pragmatic approach to hamess the power of SOA as purpose-built, easy-to-consume and easy-to-use prodpingts, while simultaneously helping to enable you to leverage the
value of your existing infrastructure value of you

The IBM WebSphere DataPower SOA Appliance family includes:

- IBM WebSphere DataPower XML

- IBM WebSphere DataPower XML

Security Gateway XS40

- IBM WebSphere DataPower

The IBM WebSphere DataPower SOA Appliance family contains $1 \mathrm{U}$ network devices that can

- Deliver robust middleware functionality in network appliance packaging that greatly simplifies

- Enhance security protection for Web services with comprehensive drop-in security functionality

- Accelerate time to deployment latency and increasing throughput of XML. Web services and SOA processing

Enhanced security: IBM WebSphere DataPower SOA Appliances provide an easy-to-install and

easy-to-maintain network appliance that can satisfy both application and network operational groups.

This announcement is provided for your information only. For additional information, contact your IBM representative,

IIM Europe, Madie East, Arica IEM is a registered trademark of Intemational Bualness Machines conporation.

supporting current and emerging Web services standards

out-of-the-box. Key support XMLISOAP firelling field XML security, data validation, XM Web services access control, service virtualization, and SSL acceleration.

Simplicity: IBM WebSphere DataPower SOA Appliances deliver common message transformation. integration and routing functions in a network device, helping to cut operational costs, reduce complexity, making on demand data integration part of the shared SOA

infrastructure, DataPower appliances are designed to be a game-changing approach for application integration and SOA.

Acceleration: IBM WebSphere DataPower SOA Appliances offer an can streamline XM and We service can streamiline XM and Web service deployments, helping lower total cost on on your assets, as you continue to purpose-built hardware devices capable of offloading overtaxed servers by processing XML Web services and other message format at wirespeed.

Key prerequisites

- Network Interface and network

cable

- Network information (IP address,

- Cor Gateway) port, keyboard, mouse

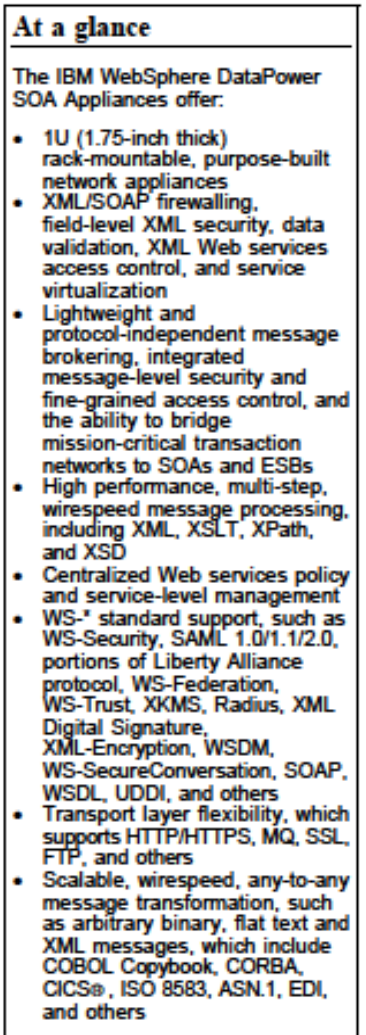

\section{At a glance}

$1 \mathrm{U}$ (1.75-inch thick)

field-level XML security, data validation, XML Web services virtualization

Lightweight and rokering, integrated and ability to bridge High performance, multi-step. wirespeed message processin

ervices polic $10 / 1 / 20$ (1) ation,

Transport layer flexibity s.ports HITP/HTTPS, MQ SSL and others

Source: https://www-01.ibm.com/common/ssi/rep_ca/3/877/ENUSZG06-0353/ENUSZG06-0353.PDF 


\section{Exhibit 6: SOA Architecture}

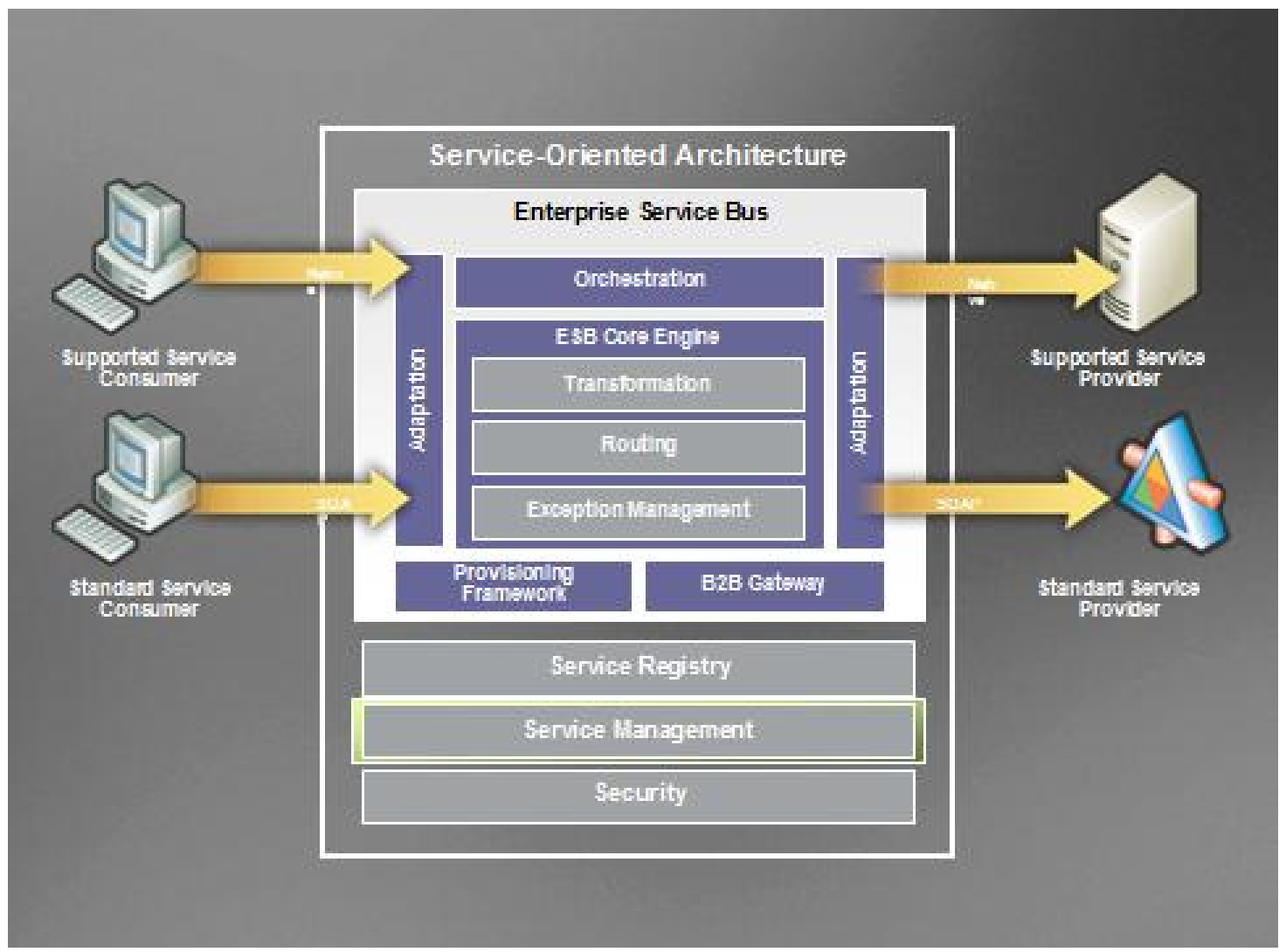

Source: https://www.slideshare.net/mikejwalker/soa-in-financial-services 
MANAKA

\section{Exhibit 7: DataPower Firmware Support Lifecycle}

$6 / 7 / 2017$

IEM Datapower Gateways FIrmware Support Ufecycle - United Kingdom

IBM DataPower Gateways Firmware Support Lifecycle

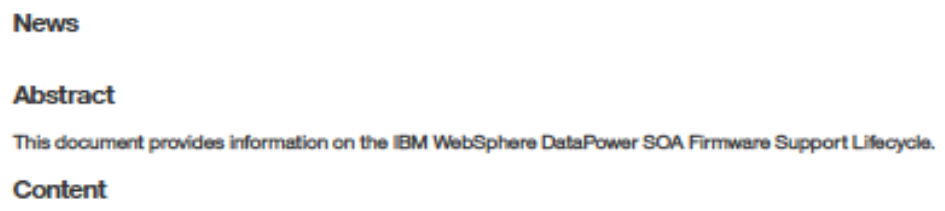

- Provide a minimum of five years device product technical support, starting at the General Availability of the new product. Beginning with the 8436 models, standard appliance service and support is available for five years from the end-of-marketing (EOM) date as described in the IBM Appliance Support Handbook

- Publish a notice of support discontinuance (end-of-service) for a product at least 12 months before the effective date For end-of-service information on physical appliances, see IBM DataPower Gateways End of Service dates.

IBM DataPower Gateways wil provide a minimum of 2 years of support for each firmware release begining at the general availabiinty (GA) of the release. IBM DataPower Gateways will continue to support the latest three levels of its firmware even if some of those frmware levels heve aged more than 2 years. We will make these releases and their latest updates avalable from our support webpege. Relaeses of the IBM DataPower Gateways firmware that are older than 2 years and are not within the three latest levels of frmware are subject to support withdrawal at any time. Customers will receive a 12-month notice of this withdrawal for planning purposes.

Note: IBM DataPower Gateways customers can use the following table to track how long their version and release of a particular frmware level will be supported. By using the information in this table, customers can effectively plan their firmware investment without ary gaps in support. Customers will receive 12 months notice prior to the end-of-service date.

\begin{tabular}{|c|c|c|c|}
\hline Pelease & GA date & End-of-service date & Comment \\
\hline 7.5 .0 & p3/2016 & Planning Information & \\
\hline 75.1 & p6/2016 & & \\
\hline 7.5 .2 & p9/2016 & & \\
\hline 2.0 & $66 / 2015$ & Planning Information & \\
\hline 7.0 & $11 / 2014$ & Planning Information & \\
\hline 70.0 & $66 / 2014$ & $09 / 30 / 2017$ & 7.0 .0 Withdrawal Announcement \\
\hline .02 (Common Criteria) & $05 / 2015$ & & \\
\hline 3.01 & $12 / 2013$ & $06 / 19 / 2016$ & 8.0.1 Withdrawal Announcement \\
\hline 3.00 & $66 / 2013$ & $06 / 19 / 2016$ & 8.0.0 Withdrawal Announcement \\
\hline 5.0 .0 & b6/2012 & $06 / 30 / 2015$ & 50.0 Withdrawal Amouncernert \\
\hline 4.0 .2 & p9/2011 & $71 / 30 / 2014$ & 40.2 Withdrawal Announcernert \\
\hline 4.0 .1 & p6/2011 & $06 / 30 / 2014$ & 40.1 Withdrawal Amouncernernt \\
\hline 4.0 .0 & p6/2011 & $09 / 30 / 2013$ & 40.0 Withdrawal Amouncernert \\
\hline 3.82 & $12 / 2010$ & $09 / 30 / 2013$ & 3.8.2 Withdrawal Amouncement \\
\hline 3.81 & p6/2010 & $12 / 31 / 2012$ & 3.8.1 Withdrawal Announcement \\
\hline 3.80 & $11 / 2009$ & $09 / 30 / 2012$ & 3.8.0 Withdrawal Announcement \\
\hline 3.7 .3 & p4/2009 & $12 / 3 \sqrt{2} 2011$ & 37.3 Withdrawal Announcernent \\
\hline 1.0 .0 & $12 / 2008$ & $12 / 3 \sqrt{2010}$ & 10.0 Announcement Archived \\
\hline 3.7 .2 & $12 / 2008$ & $12 / 3 \sqrt{2010}$ & 3.7.2 Announcement Archived \\
\hline 3.7 .1 & $08 / 2008$ & $06 / 31 / 2010$ & 37.1 Announcement Archived \\
\hline 3.7 .0 & $07 / 2008$ & $08 / 31 / 2010$ & 37.0 Announcement Archived \\
\hline 3.61 & $12 / 2007$ & $12 / 31 / 2009$ & 3.6.1 Announcement Archived \\
\hline 3.60 & $10 / 2006$ & $08 / 31 / 2009$ & 3.6.0 Announcernent Archived \\
\hline 3.5 .1 & $66 / 2006$ & $02 / 28 / 2009$ & 35.1 Announcement Archived \\
\hline
\end{tabular}

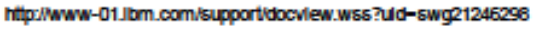

Source: http://www-01.ibm.com/support/docview.wss?uid=swg21246298 
MUMA CASE REVIEW

\title{
Exhibit 8: Woolworths' David Jones Acquisition
}

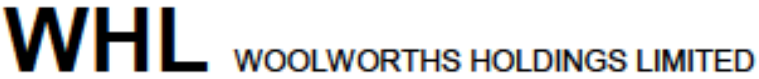

9 April 2014

\section{Woolworths South Africa to acquire David Jones to create a leading southern hemisphere retailer}

\begin{abstract}
Overview
Woolworths Holdings Limited (JSE: WHL) ('WHL') offers R21.4 billion (A\$2.1 billion) to acquire the entire issued share capital of David Jones Limited (ASX: DJS) ("David Jones") for cash consideration of AS4.00 per share.

The David Jones board of directors has unanimously resolved to recommend its shareholders vote in favour of the transaction, in the absence of a superior proposal and subject to an Independent Expert concluding that the transaction is fair and reasonable and in the best interests of David Jones shareholders.

WHL's offer price represents a $25.4 \%$ premium to the closing David Jones share price on 8 April 2014.

The combination of WHL and David Jones will create a leading southern hemisphere retailer with pro-forma combined FY13A revenue of over R51 billion (A\$5.7 billion) from 1,151 stores across 16 countries, with approximately $43 \%$ of sales generated in Australasia. ${ }^{2}$

The Combined Business

David Jones is an iconic Australian brand and occupies a similar customer positioning to Woolworths in South Africa at the premium end of the apparel business, with both businesses enjoying strong aspirational brand identities and a strong alignment of values that put the customer first, offering excellent service and quality. David Jones is one of Australia's oldest and most prominent department stores. It operates 38 department stores across Australia and owns its flagship stores in Sydney and Melbourne.

WHL has operated successfully in Australia for over 15 years through its subsidiary. Country Road Group, which operates the Country Road, Trenery. Witchery and Mimco brands.

The combination of WHL and David Jones provides significant advantages that will benefit both companies and their customers. The group will have increased scale that will drive significant efficiencies and economies through enhanced global sourcing and the ability to leverage shared seasonality and trends, improving value for the customer and overall profitability. Each business will be well equipped to compete with global retailers in their respective markets.
\end{abstract}

\footnotetext{
'As figures are comverted at the R/AS exchange rate of 9.95, the current forward exchange rate which is expected to be in IIne with the spot rate at completion

${ }^{2}$ As figures are comverted at the R/AS exchange rate of 9.12, the dally average of the exchange rate from 29/07/2012 to $27 / 07 / 2013$, being the 52 weeks period of David Jones' fnanclal year 2013.

Woolworths Holdings LImited Reg. No. 19291001986/06

Woolworths House 93 Longmarket Street Cape Town 8001 Telephone +27 214079111 Fax +27214072151

Directors: SN Susman" (Chalman) I Molr" (Group chlef executive omincer) (Australlan)

PD Bacon" (Britsh) ZBM Bassa" TA Boardman" AT Higginson"* MR Isaacs"- MU Leeming" SD Ngumenl"- AC Nissen" Sir SA Rose" (British) Z Rylands" NT Siwendu" Group secretary T Sishuba-Mashego
}

"Non-executive "Execultive

Source: http://www.woolworthsholdings.co.za/whl_mini_2014/pdf/press_release_final.pdf 


\section{Exhibit 9: Woolworths Values and Description}

Table 1: Woolworths Values and Description

\begin{tabular}{|l|l|}
\hline Values & Description \\
\hline Quality and style & Deliver the best \\
\hline Value & A simple and fair deal \\
\hline Service & We always think customer \\
\hline Innovation & Discover the difference \\
\hline Integrity & Doing what you say you will do \\
\hline Energy & Be passionate and deliver \\
\hline Sustainability & Build for better future \\
\hline
\end{tabular}

Source: Developed by case writer. 
Exhibit 10: Woolworths Holdings LTD Groups

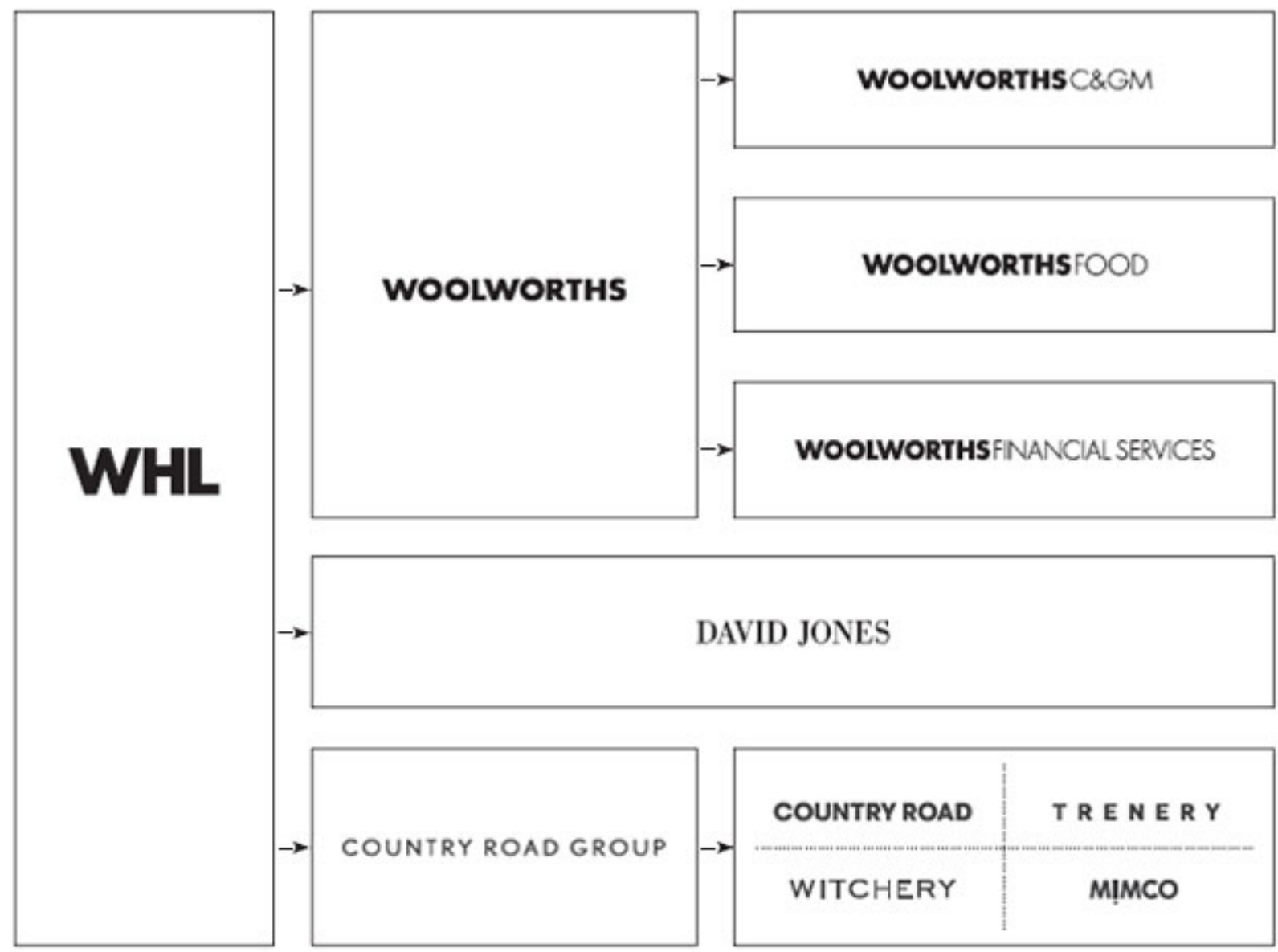

Source: Developed by case writer. 


\section{Exhibit 11: AIT Decision making Hierarchy}

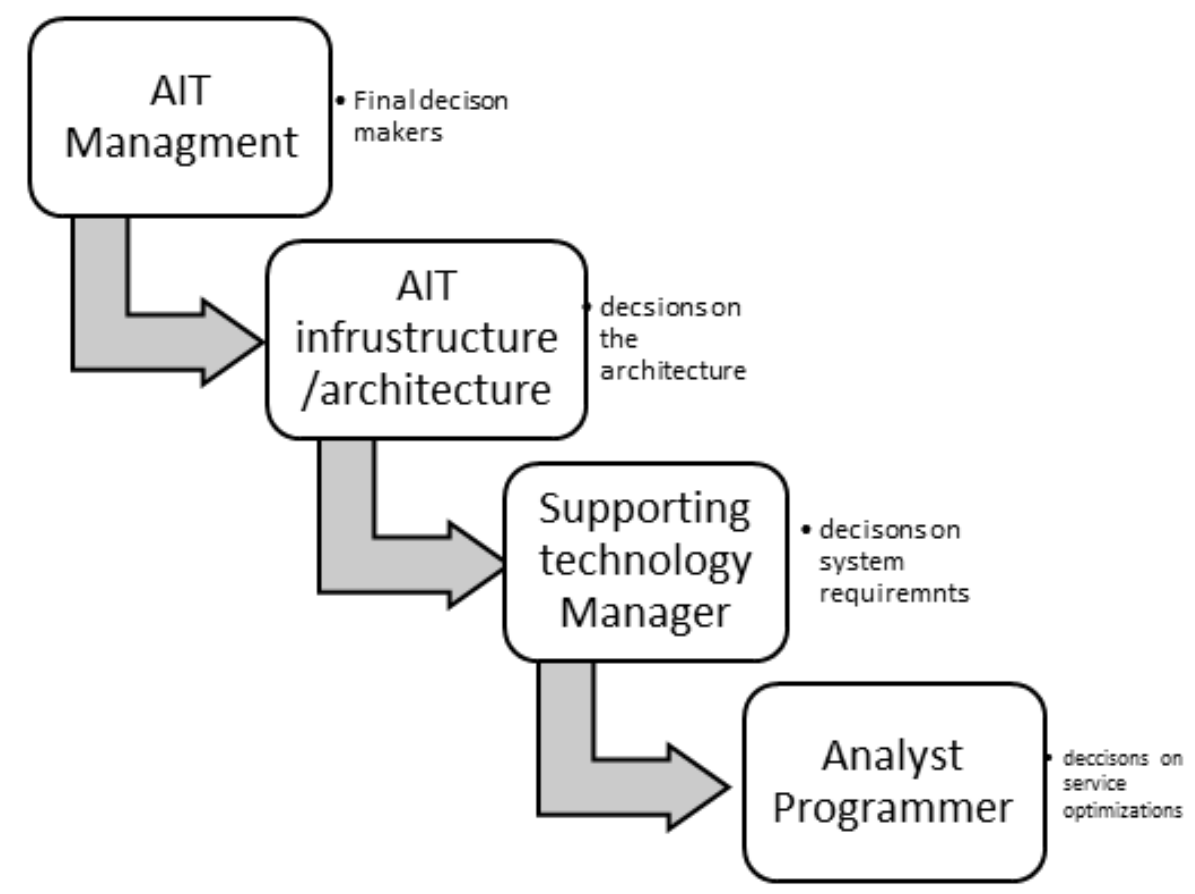

Figure 1: AIT Decision making Hierarchy 